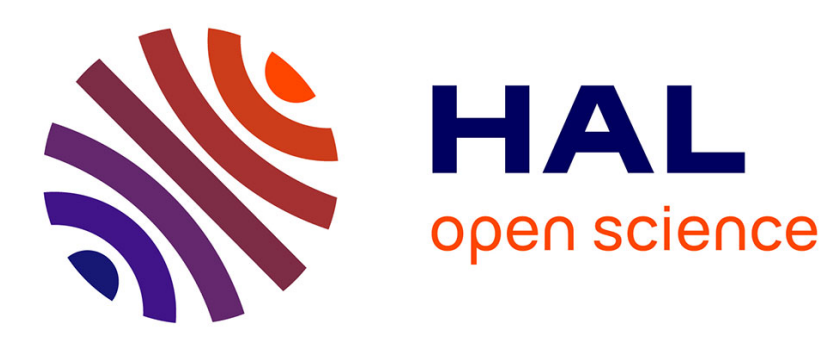

\title{
Holisme, anatomicité et hiérarchie
}

Fabrice Pataut

\section{To cite this version:}

Fabrice Pataut. Holisme, anatomicité et hiérarchie. Archives de Philosophie, 2008, 71 (4), pp.599-607. 10.3917/aphi.714.0599 . halshs-00791192

\section{HAL Id: halshs-00791192 \\ https://shs.hal.science/halshs-00791192}

Submitted on 17 Nov 2020

HAL is a multi-disciplinary open access archive for the deposit and dissemination of scientific research documents, whether they are published or not. The documents may come from teaching and research institutions in France or abroad, or from public or private research centers.
L'archive ouverte pluridisciplinaire HAL, est destinée au dépôt et à la diffusion de documents scientifiques de niveau recherche, publiés ou non, émanant des établissements d'enseignement et de recherche français ou étrangers, des laboratoires publics ou privés. 
[Version modifiée de l'article publié in Archives de Philosophie, cahier 71-4, hiver, pp. 599-607.]

\title{
HOLISME, ANATOMICITÉ ET HIÉRARCHIE
}

\author{
Fabrice Pataut \\ Institut d'Histoire et de Philosophie des Sciences et des Techniques \\ (IHPST), Paris
}

\begin{abstract}
Résumé
Selon le holisme sémantique, les propriétés sémantiques sont par nature anatomiques, c'est-à-dire intrinsèquement collectives. Selon le holisme de l'interprétation, la signification ou le contenu sont attribués collectivement. La thèse de constitution holistique de la signification peut être raisonnablement défendue à l'aide d'une contrainte de molécularité dont le rôle est d'introduire une hiérarchie dans la complexité logique des phrases, autrement dit un ordre ou une articulation dans le langage qui fasse droit à une condition finitaire d'anatomicité.
\end{abstract}

\section{Mots-clés}

Complexité logique, holisme, molécularisme, signification ; Davidson (D.), Dummett (M.), Fodor (J.).

\begin{abstract}
According to semantic holism, semantic properties are anatomic by nature, i.e. intrinsically collective. According to interpretation holism, meaning, or content, is collectively attributed. The thesis that meaning is holistically constituted may be defended provided we take into account a molecularity requirement. The requirement's rôle is to introduce order or articulation in the langage, via a hierarchy of logical complexity that pays due attention to a finitary condition of anatomicity.
\end{abstract}

\section{Key words}

Holism, logical complexity, meaning, molecularism ; Davidson (D.), Dummett (M.), Fodor (J.). 
1. On pourrait aisément appliquer au cas du holisme une remarque que Paul Horwich (Horwich 1982: 181) a faite de manière très pertinente à propos du réalisme, à savoir que la conduite du débat (pour nous, ici : faut-il, oui ou non, être holiste ?) est gênée par une aversion systématique à formuler la doctrine explicitement, de manière que des positions différentes ont été tour à tour identifiées avec le holisme et que l'équivoque et le quiproquo sont assez systématiquement de mise dans la présentation des arguments et des contrearguments. Par parité, la situation n'est pas toujours plus enviable du côté du molécularisme qu'on lui oppose, généralement en porte-à-faux.

L'idée, ici, est simplement de défricher le terrain, de déterminer ce qui peut être fait pour défendre contre certaines objections l'idée selon laquelle, en première approximation, la signification des phrases complètes d'un langage dépend de la signification d'autres phrases complètes du même langage, d'arriver à donner les détails de cette dépendance de manière à la rendre la plus spécifique possible sans risquer, à un moment ou à un autre, de trahir cette idée directrice.

Il y a une grande latitude dans la manière dont on comprend la dépendance en question : tantôt en termes de la place qu'occupent les phrases auxquelles on entend attribuer une signification dans la structure constituée par le langage auquel elles appartiennent pris comme un tout, tantôt en termes de leurs interconnections inférentielles, tantôt en termes de compréhension ou d'usage correct du dit langage pris dans sa totalité (autrement dit, de la totalité de nos pratiques linguistiques), tantôt encore en termes du lien d'inextricabilité qui s'établit entre signification et théorie, signification et schème conceptuel, signification et croyances (justifiées ou non). On la comprend même parfois plus en termes de ce que l'une ou l'autre de ces positions est censée impliquer, que, stricto sensu, ces positions elles-mêmes, qu'elles soient prises isolément ou collectivemment, par exemple : l'impossibilité d'attribuer un contenu à chaque phrase du langage qui rende compte de la diversité des moyens dont nous 
pouvons disposer pour établir sa vérité (Dummett 2001: 75), ou bien l'impossibilité de représenter systématiquement les liens déductifs entre les phrases que nous tenons pour vraies (Dummett loc. cit.).

Il est bien évidemment essentiel de distinguer ces positions le plus finement possible et de déterminer ce à quoi nous engage chacune séparément des autres. Mais avant d'aller dans le détail de ce qui constitue cette dépendance, il est important de comprendre à quoi nous engage, de manière générale, une thèse de dépendance.

Si nous tenons à nous exprimer en termes de possession ou d'attribution de propriété, nous dirons qu'en soutenant une thèse de dépendance, nous affirmons que quelque chose possède une propriété, ou que nous pouvons légitimement en attribuer une à un objet, si et seulement si une certaine condition est satisfaite, et que nous énonçons par là-même les conditions minimales de la possession ou de l'attribution.

Les différentes manières d'expliciter la nature de la dépendance en question, dont nous venons de donner un très bref aperçu, laissent intact ce que nous voudrons mettre ici à jour, à savoir qu'une phrase $p$ d'un langage $L$ a une signification si et seulement si il existe au moins une autre phrase $q$ de $L$ qui a également une signification.

Pour reprendre la terminologie de Fodor \& Lepore 1992, la propriété qu'ont les phrases d'avoir une signification est une propriété anatomique (par opposition à une propriété atomique [atomistic ou punctate] qui, elle, peut en principe être instantiée par un seul objet): une phrase ne peut avoir de signification qu'à la condition qu'au moins une autre en ait également une. La signification n'est pas une propriété qu'une phrase complète peut avoir isolément.

Si nous continuons dans cette veine, nous ferons remarquer, avec Fodor et Lepore (op. cit. : 2-3), que certaines propriétés anatomiques sont holistiques, par exemple la propriété d'être un entier naturel conçue par référence à la relation 
« être le successeur de » : si quelque chose est un entier naturel, alors il existe non seulement au moins un autre objet qui est un entier naturel, à savoir son successeur (et cela fait de la propriété en cause une propriété anatomique), mais, qui plus est, beaucoup d'autres et même une infinité de tels objets (ce qui fait de la propriété anatomique une propriété holistique.)

En parlant comme nous l'avons fait, nous avons traité les significations comme des propriétés que les phrases possèdent. Comme le $\mathrm{McX}$ de Quine $1980 a$, nous les avons traitées comme des entités abstraites. Nous pourrions procéder autrement : refuser, comme Quine, d'analyser le fait qu'une phrase soit pourvue de sens (sa meaningfulness) en termes de possession d'une entité de ce genre et choisir de l'analyser directement (Quine op. cit. : 11) en termes de ce que font les locuteurs en présence de son élocution et d'autres élocutions similaires et appropriées. Nous exprimerons alors le holisme sémantique en terme de comportement ou de disposition au comportement en commençant par faire valoir, pour dire les choses très rapidement, que ce que les locuteurs font, ou ont tendance à faire, ou sont disposés à faire en présence de l'élocution particulière d'une phrase, est déterminé par ce qu'ils font ou ont tendance à faire en présence d'autres élocutions d'autres phrases, et que ce qui lie ces phrases entre elles est précisément fonction de leur comportement, ou de leur disposition ou tendance au comportement.

En faisant cela, nous ferons sans nul doute dépendre le holisme sémantique du holisme de la pratique de l'interprète et, du coup, de la thèse d'inextricabilité. Quoi qu'il en soit de la légitimité de cette inférence du holisme de la pratique interprétative au holisme sémantique, il est raisonnable de considérer que la question de savoir si la signification d'une phrase dépend ou non de la signification d'autres phrases est indépendante de la question de savoir quelle analyse de la signification il vaut mieux choisir: l'analyse en termes de propriétés ou d'entités abstraites (et nous prenons ici les expressions « propriété » et «entité abstraite » en un sens littéral et pas seulement comme 
une simple façon commode de parler), ou bien l'analyse en termes comportementalistes ou en termes de stratégie interprétative à la Davidson. Ce qui compte, tout au moins en ce qui concerne la caractérisation minimale et provisoire du holisme que nous avons retenue, est que, dans un cas comme dans l'autre, nous devons faire l'économie de la notion de contenu individuel et que nous savons, au moins par contraste avec le cas de l'anatomicité, qu'un contenu est individuel exactement au cas où il peut être déterminé, fixé ou identifié phrase par phrase, autrement dit, en prenant uniquement en compte les occurrences internes de leurs parties constituantes, comme si aucune autre phrase n'existait ou n'était exprimable dans le langage et, a fortiori, comme si aucune ne contenait des occurrences (externes, pour le coup) de ces constituantslà.

$\mathrm{Ou}$ encore, pour reprendre la distinction de Quine entre grammaire et lexicographie (voir Quine 1980b: 49), si nous adoptons la position du grammarien qui cherche quelles formes linguistiques ont une signification, ou si, comme le lexicographe, nous cherchons lesquelles sont synonymes, nous travaillerons sans jamais nous en remettre à cette notion de contenu individuel, en ayant par ailleurs évacué toute notion réifiée ou hypostasiée de signification. Et si nous tenons à défendre ce que Fodor et Lepore jugent être une impasse, nous nous engagerons dans une théorie des conditions métaphysiquement nécessaires de la possession de signification ou de contenu par tout ce qui peut légitimement avoir cette propriété, soit de manière directe, soit de manière dérivée : les phrases, les énoncés, les élocutions, les propositions, les croyances, en en faisant également l'économie. Nous ferons, dans ce cas-là, une théorie des conditions d'instantiation d'une propriété anatomique.

Dans le premier cas, nous aurons abandonné une notion non hypostasiée de contenu individuel; dans le deuxième, nous aurons abandonné la notion de propriété atomique. Dans les deux cas, nous chercherons à déterminer, à fixer, ou à attribuer le contenu ou la signification des phrases complètes en prenant en 
compte la signification des occurrences internes et des occurrences externes de leurs parties constituantes.

2. Fodor et Lepore (loc. cit.) considèrent qu'il est inutile, pour cerner correctement le caractère (prétendument, selon eux) holistique des propriétés sémantiques (avoir une signification, avoir un contenu, exprimer une proposition, avoir un référent, être vérifiable effectivement ou en principe, etc...), de chercher à préciser combien d'autres objets doivent également avoir ces propriétés si un objet les $\mathrm{a}$, ou à distinguer les cas où un nombre fini doivent l'avoir de ceux où un nombre infini doivent l'avoir, de sorte que le caractère nécessairement ou intrinsèquemment collectif de la possession suffit.

Du côté de la stratégie interprétative, Davidson considère que le caractère infinitaire est une condition nécessaire du holisme de l'attribution de croyances, autrement dit du holisme qui règle l'assignation d'une signification aux phrases prononcées par nos interlocuteurs dans les situations et les contextes appropriés :

«Quiconque peut interpréter une élocution de la phrase française «Le revolver est chargé » doit avoir un grand nombre de croyances, et ces croyances doivent être tout à fait comme celles que quelqu'un doit avoir s'il pense que le revolver est chargé. Nous pouvons supposer que l'interprète doit croire qu'un revolver est une arme et qu'il s'agit d'un objet physique plus ou moins persistant (enduring). Il n'y a probablement pas de liste déterminée (definite) de choses qui doivent être crues par quelqu'un qui comprend la phrase «Le revolver est chargé », mais il est nécessaire qu'il y ait un nombre infini de croyances imbriquées (but it is necessary that there be endless interlocked beliefs). »

Davidson 1985 : 158

(Comme le remarque Montminy (voir Montminy 2005), Davidson énonce ici les conditions de constitution du mental - autrement dit les conditions de constitution de ce que l'interprète doit assigner aux locuteurs pour effectuer son travail d'interprétation, à savoir une psychologie - et non pas les normes 
gouvernant les êtres bénéficiant d'une vie mentale. Montminy pourrait même faire remarquer à l'appui de sa lecture modale de «doit» dans «doit avoir un grand nombre de croyances », qu'un holisme flexible, pour lequel il n'y a pas de liste fixe de croyances qu'il faudrait attribuer à un locuteur pour qu'on puisse finalement lui attribuer une croyance particulière, ne peut légitimer le «doit » normatif. Ou alors, si le «doit» est normatif, il l'est seulement pour les interprètes, au sens où la condition holistique règle la manière dont ceux-ci - les interprètes - doivent recourir aux concepts intentionnels dans la pratique interprétative.)

Il s'agit bien ici du holisme de l'attribution d'entités qui sont nécessaires à l'assignation de signification aux phrases, à savoir les croyances, et non pas du holisme sémantique lui-même, autrement dit du holisme du contenu ou de la signification des phrases et des énoncés (c'est-à-dire des phrases prononcées à telle ou telle occasion, dans tel ou tel contexte). On peut faire valoir à cette occasion ce que Fodor 1987: 61-62 fait valoir contre Stich 1983: 54 et suivantes, à savoir que la sensibilité d'une attribution de croyance à une autre attribution de croyance n'implique nullement la sensibilité du contenu de la première à celui de la deuxième, et qu'il en est de même de la sensibilité des assignations de signification aux phrases vis-vis des significations correspondantes.

Si cette position est correcte, et pour reprendre ici la terminologie de Fodor, on peut justifier l'attribution d'une croyance à un individu par le recours aux liens épistémiques sans pour autant avoir à soutenir que le contenu de la croyance ainsi attribuée est individué anatomiquement, c'est-à-dire par un autre contenu, notamment celui de la croyance assurant le lien épistémique, autrement dit par une autre proposition dont la valeur de vérité est pertinente pour la détermination de la valeur de vérité de la croyance exprimée. De même pour l'attribution de signification à une phrase, par le recours à d'autres attributions, relativement aux conditions d'individuation de cette signification. 
Supposons qu'un interprète attribue à un locuteur la croyance que le revolver est chargé parce qu'il dispose par ailleurs d'une évidence en faveur de la croyance du locuteur qu'un revolver est une arme (plutôt le genre de situation envisagée par Fodor). Ou bien supposons qu'il lui attribue cette croyance parce qu'il ne peut mieux expliquer l'interprétation correcte de la phrase « Le revolver est chargé » par le locuteur qu'en lui attribuant les croyances que lui aussi, l'interpréte, possède sur le chapitre des armes (plutôt le genre de situation envisagée par Davidson par l'application du principe de correspondance, qui recommande à l'interprète d'attribuer à l'agent des croyances qui maximisent leur accord (voir Davidson 1991 : 158)). Dans un cas comme dans l'autre, rien de sémantique ne s'ensuit quant à la signification de la phrase « Le revolver est chargé », autrement dit quant à la détermination de ses conditions de vérité et de celles de ses élocutions particulières. Il est possible qu'une signification lui soit attribuée de manière anatomique sans pour autant que ses conditions de vérité soient déterminées de cette même manière, autrement dit sans qu'elles dépendent pour autant des conditions de vérité des autres phrases du langage que l'interprète a prises en compte et, a fortiori, de celles que l'interprète n'a pas prises en compte.

Si rien de sémantique quant au contenu de la croyance que le revolver est chargé et quant à la signification de la phrase française qui l'exprime, à savoir «Le revolver est chargé », ne suit de ces pratiques interprétatives, nous le devons, non pas à la flexibilité du holisme, qui fait que locuteur et interprète ne partagent pas nécessairement les mêmes croyances sur les revolvers et ne tiennent pas exactement ou systématiquement les mêmes phrases afférantes pour vraies, mais à une distinction catégorique ou de nature entre deux formes radicalement étrangères de holisme : celui du contenu ou de la signification, et celui de l'attribution. Le premier affirme qu'il n'y a de contenu que collectif. Le deuxième que nous devons supposer qu'autrui ne saisit ou ne croie le contenu que collectivement. 
3. La question importante est maintenant de savoir si, indépendamment du cas du holisme de la pratique interprétative et des positions connexes qui servent à le caractériser comme, typiquement, la thèse d'inextricabilité, le partisan du holisme du contenu peut, lui, à la manière de Fodor et Lepore, juger que le caractère fini ou, au contraire, infini de l'ensemble des phrases qui doivent avoir une signification pour qu'une phrase particulière en ait une, n'est nullement pertinent pour sa caractérisation du holisme, ou s'il lui faut au contraire défendre contre certaines objections un choix qu'il devra faire sur ce point particulier.

Nous cherchons à préciser l'idée schématique minimale et incomplète de départ en déterminant quelles conditions doivent être réalisées pour que la signification d'une phrase soit holistiquement constituée en plus de la :

(1) Condition d'anatomicité: la signification de $p$ dépend de la signification d'au moins une autre phrase $q$.

Deux possibilités se présentent :

(2) Condition infinitaire: la signification de $p$ dépend de la signification d'un ensemble infini d'autres phrases $\{q, r, s, \ldots\}$

(3) Condition finitaire : la signification de $p$ dépend de la signification d'un ensemble fini d'autres phrases $\{q, r\}$

(3a) pas assez petit pour qu'on puisse identifier, compter et inspecter ses membres en un temps fini.

(3b) assez petit pour qu'on puisse identifier, compter et inspecter ses membres en un temps fini.

Une objection contre (2) et (3a) consiste à faire valoir que la satisfaction de l'une ou l'autre de ces conditions est en conflit avec l'injonction de manifestabilité conçue à la Dummett : dans un cas comme dans l'autre, il y a trop de phrases à prendre en compte pour qu'il soit humainement possible 
d'identifier toutes celles qui peuvent servir à déterminer ou à fixer la signification d'une phrase en particulier. Qu'il y en ait, autrement dit qu'il faille en prendre en compte ne pose, de ce point de vue, aucun problème particulier. La condition d'anatomicité est au contraire bienvenue: pour satisfaire l'injonction de manifestabilité de la saisie de la signification d'une phrase dans l'hypothèse où celle-ci est déterminée par ses conditions de justifiabilité ou d'assertabilité, il faut pouvoir déterminer quelles autres phrases justifient son assertion, rendre compte de la diversité des moyens dont nous disposons pour établir sa vérité (voir, sur ce point, Bilgrami 1986 : section III).

L'aspect déterminant de l'objection dummettienne porte non pas tant sur l'étendue, la taille ou le caractère non inspectable du langage présupposé par la doctrine holiste, caractéristiques qui seraient jugées fautives parce qu'incompatibles avec l'idée selon laquelle la saisie du sens doit être exhaustivement manifestable dans la pratique ou l'usage, que sur le caractère informe de sa structure - et même, sur son absence complète de structure - dans l'hypothèse où aucune hiérarchie logique n'est imposée ou prise en compte, même dans le cas de figure (3b). Cette objection, ou cet aspect de l'objection dummettienne, fait valoir qu'à moins d'introduire un ordre et une hiérarchie dans l'ensemble des phrases qui peuvent servir à déterminer la signification d'une phrase particulière, il est impossible, non pas tant de donner une représentation de sa signification, que d'en donner une représentation articulée. Il devient impossible de savoir quelle partie de la structure, ou quel aspect de la pratique doit être tenue pour responsable de sa détermination.

On peut pallier à cette difficulté en introduisant une exigence de molécularité. Il est sans doute tout aussi malaisé de trouver une expression claire et univoque du molécularisme qu'il est malaisé de trouver une expression claire et univoque du holisme. Son expression la plus convaincante et la plus claire est peut-être, après tout, celle-ci : la signification d'une phrase dépend uniquement de la signification des occurrences internes de ses parties constituantes (ce qui revient 
ni plus ni moins à affirmer la thèse de compositionnalité) et de la signification des occurrences externes de ces mêmes parties constituantes dans des phrases de même complexité logique ou de complexité logique inférieure.

L'idée selon laquelle la signification d'une phrase ne peut dépendre de la signification de phrases logiquement plus complexes introduit un ordre et une hiérarchie qui font entièrement droit à la condition d'anatomicité et à la condition finitaire. On commence typiquement avec un langage ne contenant que des phrases atomiques et on lui ajoute des constantes logiques en imposant une condition d'extension conservative. Le nouveau langage est une extension conservative du langage originel si et seulement les phrases assertables du langage originel le restent dans le langage augmenté des constantes logiques.

Est-on vraiment en train de défendre le holisme en proposant cela ? Cela dépend, au moins en partie, du genre de théorie de la signification qu'on aura choisie pour les expressions non logiques du langage. Il y a bien évidemment le cas de la complexité logique explicite, prise en compte ici par l'exigence d'extension conservative que je viens de caractériser très brièvement, mais il y a également d'autres formes de complexité à prendre en considération, qui ne recouvrent pas le cas de la complexité logique et ne coïncident pas avec lui. Il y a notamment le cas de la complexité du vocabulaire non logique, ou de la hiérarchie lexicale, par exemple pour les termes d'espèces naturelle. Là aussi la condition d'anatomicité est à prendre en compte. L'adoption du molécularisme pour les constantes logiques laisse le choix entièrement ouvert en ce qui concerne l'adoption ou le rejet d'une forme particulière de molécularisme pour les expressions non logiques du langage. Là aussi, il sera utile d'envisager séparément les différents ingrédients qui peuvent raisonnablement servir à donner un contenu précis à la doctrine du holisme sémantique. Il faudra notamment déterminer si nous pouvons espérer trouver un analogue non formel $\mathrm{du}$ molécularisme pour les expressions non logiques qui entrent dans la composition des phrases complètes. Là aussi, nous pouvons au moins tenter 
d'éviter ce que certains ont tenu à appeler le « holisme global », autrement dit la position selon laquelle nous devrions comprendre ou saisir la totalité du lexique ou des explications verbales relatives aux expressions constituantes des phrases d'un langage pour saisir la signification d'une seule de ses phrases. Une défense appropriée du holisme sémantique dépend en partie des résultats que nous pourrons établir en orientant la recherche dans cette direction. 


\section{RÉFÉRENCES}

BILGRAMI (Akeel), 1986, «Meaning, Holism and Use», Truth and Interpretation - Perspectives on the Philosophy of Donald Davidson, E. LePore, ed., Basil Blackwell, Oxford, pp. 101-122.

DAVIDSON (Donald), 1985, « Thought and Talk (1975)», Inquiries into Truth and Interpretation (éd. corrigée), Clarendon Press, Oxford, pp. 155-170.

- 1991, «Three Varieties of Knowledge », A. J. Ayer : Memorial Essays, A. P. Griffiths, dir., Cambridge U.P., pp. 153-166.

DUMMETT (Michael), 2001, «La justification de la déduction », Philosophie, (I) : $\mathrm{n}^{\circ} 71$, pp. 58-79; (II) : $\mathrm{n}^{\circ} 72$, pp. 36-54.

FODOR (Jerry), 1987, Psychosemantics: The Problem of Meaning in the Philosophy of Mind, The M.I.T. Press, Cambridge, Mass.

FODOR (Jerry) \& LEPORE (Ernest), 1992, Holism - A Shopper's Guide, Basil Blackwell, Oxford and Cambridge.

HORWICH (Paul), 1982, «Three Forms of Realism », Synthese, vol. 51, n² : Realism, Part I, pp. 181-201.

MONTMINY (Martin), 2005, «Interprétation et interprétationnismes », Philosophiques, Vol. 32, n 1, pp.3-17.

QUINE (Willard Van Orman), 1980a, «On What There Is », From a Logical Point of View -9 Logico-Philosophical Essays, Harvard U.P. ( $3^{\text {rd }}$ edition), pp. 1-19.

-1980b, «Two Dogmas of Empiricism », From a Logical Point of View - 9 Logico-Philosophical Essays, Harvard U.P. (3 ${ }^{\text {rd }}$ edition), pp. 20-46.

- 1980c, «The Problem of Meaning in Linguistics », From a Logical Point of View - 9 Logico-Philosophical Essays, Harvard U.P. (3 ${ }^{\text {rd }}$ edition), pp. 47-64.

STICH (Stephen), 1983, From Folk Psychology to Cognitive Science, The M.I.T. Press, Cambridge, Mass. 